\title{
An Implicit Method for Numerical Solution of Second Order Singular Initial Value Problems
}

\author{
Mohammad Kamrul Hasan", Mohammad Suzan Ahamed, Mohammad Ashraful Huq, \\ Mohammad Shamsul Alam and Mohammad Bellal Hossain
}

Department of Mathematics, Rajshahi University of Engineering and Technology, Rajshahi-6204, Bangladesh

\begin{abstract}
Recently an implicit method has been presented for solving first order singular initial value problem. The method is extended to solve second or higher order problems having a singular point. The method presents more correct result than those obtained by the implicit Euler and second order implicit Runge-Kutta (RK2) methods. The method is illustrated by suitable examples.
\end{abstract}

Keywords: Emden-Fowler Type Equation, Implicit Runge-Kutta Method, Lane-Emden Type Equation, Numerical Solution, Singular Initial Value Problems.

\section{INTRODUCTION}

In the recent years, the study of singular initial value problems modeled by the second order ordinary differential equations has attracted the attention of many mathematicians and physicists. Such a problem is expressed by Lane-Emden or Emden-Fowler type differential equation. The noted equations are important in the theory of stellar structure, thermal behavior of a spherical cloud of gas, isothermal gas spheres and thermionic currents [1-3].

Some analytical techniques were presented to solve Lane-Emden equations. Most of them were developed based on power series or perturbation. Wazwaz [4-7] has presented series and exact solution to Lane-Emden and Emden-Fowler type problems based on Adomain decomposition and modified Adomain decomposition methods. Hasan and Zhu $[8,9]$ have solved such a singular initial value problem by the Taylor series and modified Adomain decomposition methods. Gupta and Sharma [10] have also used the Taylor series method to solve Lane-Emden and Emden-Eowler equations. Demir and Süngü [11] have presented approximate and analytic solutions of Emden-Fowler equation and Mukherjee et al. [12] the Lane-Emden equation by the same method i.e., differential transform method. However the determination of solutions by these methods is laborious.

A few classical numerical methods can be used to solve singular initial value problem such as implicit Euler method, implicit Runge-Kutta method and implicit midpoint method etc. Koch et al. $[13,14]$ evaluated the approximate solution of the singular initial value problems by implicit Euler method and finally used an acceleration technique known as

*Address correspondence to this author at the Department of Mathematics, Rajshahi University of Engineering and Technology, Rajshahi-6204, Bangladesh; Tel: +88-0721-750349; E-mail: mkh2502@yahoo.com the Iterated Defect Correction to improve the approximations. Benko et al. also [15] evaluated singular initial value problems of Lane-Emden type equations by implicit Euler method. The second order implicit RungeKutta method is a higher order solver than the implicit Euler method for solving singular initial value problems. Lakestani and Saray [16] solved Emden-Fowler type equations numerically using Legendre scaling functions. This method consists of expanding the required approximate solution as the elements of Legendre scaling functions. Using the operational matrix of integration, the problem will be reduced to a set of algebraic equations. But utilization of this method is cumbersome.

Recently, Hasan et al. [17] derived an implicit method for solving first order singular initial value problems, which give more accurate solution than the implicit Euler method and the second order implicit Runge-Kutta (RK2) method. In this article this method is extended to solve second order problems.

\section{DERIVATION OF THE PRESENT METHOD}

First we derive the present method for solving first order singular initial value problems and then the method is extended for second order singular initial value problems.

\subsection{For First Order Singular Initial Value Problems}

Recently, Huq et al. [18] derived a formula for evaluating definite integral having an initial singular point at $x=x_{0}$ in the form as

$\int_{x_{0}}^{x_{0}+3 h} \varphi(x) d x=\frac{3 h}{4}\left[3 \varphi\left(x_{0}+h\right)+\varphi\left(x_{0}+3 h\right)\right]$

Based on formula (1), an implicit method has been proposed for solving first order singular initial value problems 
$y^{\prime}(x)=f(x, y), \quad y\left(x_{0}\right)=y_{0}$

having the initial singular point at $x=x_{0}$ in the form as [19]

$y_{1}=y_{0}+\frac{3 h}{4}\left[3 f\left(x_{0}+h, y_{0}\right)+f\left(x_{0}+3 h, y_{1}\right)\right]$

For the next steps the formula has been written as

$y_{i+1}=y_{i-1}+\frac{3 h}{4}\left[3 f\left(x_{i}, y_{i}\right)+f\left(x_{i+1}, y_{i+1}\right)\right] ; i \geq 1$

where, $x_{i+1}=x_{i}+2^{i} .3 h$

Since this formula was derived for unequal interval as $h, 3 h, 2.3 h, 2^{2} .3 h, 2^{3} .3 h, \cdots$. In this regard step size as well as error gradually increased. To avoid this difficulty the formula (3b) has been modified as [17]

$y_{i+1}=y_{i}+\frac{h}{4}\left[3 f\left(x_{i}+h / 3,\left(y_{i}+\left(y_{i+1}-y_{i}\right) / 3\right)\right)+f\left(x_{i+1}, y_{i+1}\right)\right] ;$

$i=0,1,2, \ldots$

where, $x_{i+1}=x_{i}+h$

It is obvious that Eq. (4) is an algebraic equation of unknown $y_{i+1}$ and can solve by a numerical method. To compare the present method to other classical methods such as the second order implicit Runge-Kutta (RK2) method and the implicit Euler method are given in equations (5) and (6) respectively.

$y_{i+1}=y_{i}+k ; \quad i=0,1,2, \ldots$

where $k=h f\left(x_{i}+h / 2, y_{i}+k / 2\right)$

and $y_{i+1}=y_{i}+h f\left(x_{i}+h, y_{i+1}\right) ; \quad i=0,1,2, \ldots$

The truncation error of the present method (i.e., Eq. (4)), the second order implicit Runge-Kutta (RK2) method and implicit Euler method are $\mathrm{O}\left(h^{4}\right), \mathrm{O}\left(h^{3}\right)$ and $\mathrm{O}\left(h^{2}\right)$ respectively.

\subsection{For Second Order Singular Initial Value Problems}

Let us consider a second order singular initial value problem of the form [5]

$y^{\prime \prime}+\frac{2}{x} y^{\prime}+f(x, y)=g(x), \quad 0<x \leq 1, \quad y(0)=A, y^{\prime}(0)=B$

where $A$ and $B$ are constants, $f(x, y)$ is a continuous real valued function and $g(x) \in C[0,1]$. Now equation (7) can be transformed into two first order initial value problems, one is non-singular and other is singular as

$y^{\prime}=z=f_{1}(x, y)$

$z^{\prime}=-\frac{2}{x} z-f(x, y)+g(x)=f_{2}(x, y, z)$

where $y(0)=A, z(0)=B$, and $y=y, y^{\prime}=z$.

According to the present method (i.e. Eq. (4)) the approximate solutions of the equations (8a) and (8b) are $\left.y_{i+1}=y_{i}+\frac{h}{4}\left[3\left(z_{i}+\left(z_{i+1}-z_{i}\right) / 3\right)+z_{i+1}\right)\right]$

$z_{i+1}=z_{i}+\frac{h}{4}\left[3\left\{\begin{array}{l}-\frac{2}{\left(x_{0}+h / 3\right)}\left(z_{i}+\left(z_{i+1}-z_{i}\right) / 3\right)- \\ f\left(x_{0}+h / 3,\left(y_{i}+\left(y_{i+1}-y_{i}\right) / 3\right)\right)+g\left(x_{0}+h / 3\right)\end{array}\right\}\right.$

$\left.+\left(-\frac{2}{\left(x_{0}+h\right)} z_{i+1}-f\left(x_{0}+h, y_{i+1}\right)+g\left(x_{0}+h\right)\right)\right] ; i=0,1,2, \ldots$

It is obvious that equations (9a) and (9b) is a system of equations for two unknown $y_{i+1}$ and $z_{i+1}$ and can be solved by Newton-Raphson method.

\section{STABILITY OF THE PRESENT METHOD}

Consider a scalar test equation

$y^{\prime}=\lambda y, \quad \lambda \in C, \operatorname{Re}(\lambda)<0$

Appling (4) to the test equation with $y^{\prime}=\lambda y$ and $z=\lambda h$ yields

$y_{i+1}=R(z) y_{i}$

where, $R(z)=(1+z / 2) /(1-z / 2)$ is the stability function of the present method.

For $\lambda<0$, then $|R(z)|<1$ for any $h>0$. Since $z$ is imaginary, the present method is absolutely stable in the entire negative half of the complex $z$ plane. The region of absolute stability is the set of all complex $z$ where $|R(z)| \leq 1$ . A numerical method is said to be A-stable if its stability region contains $C^{-}$, the non-positive half-plane $\{z=\lambda h \in C: \operatorname{Re}(z)<0\}$. So the present method is A-stable. The stability region of the present method is given in Fig. (1).

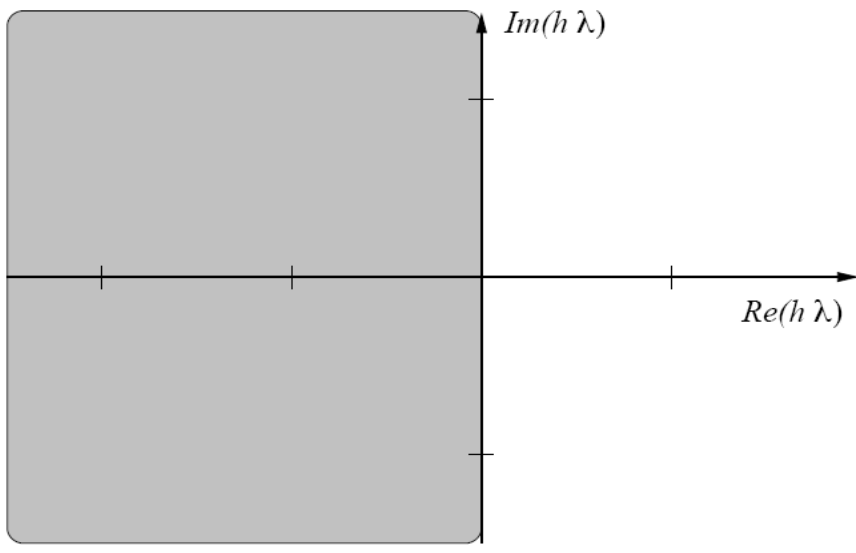

Fig. (1). Stability region of the present method.

\section{EXAMPLES}

In this section we compare the present method with the second order implicit Runge-Kutta (RK2) and the implicit Euler methods for solving following model equations.

Example 1: Consider a second order linear, nonhomogeneous Lane-Emden equation [9] 
(a)

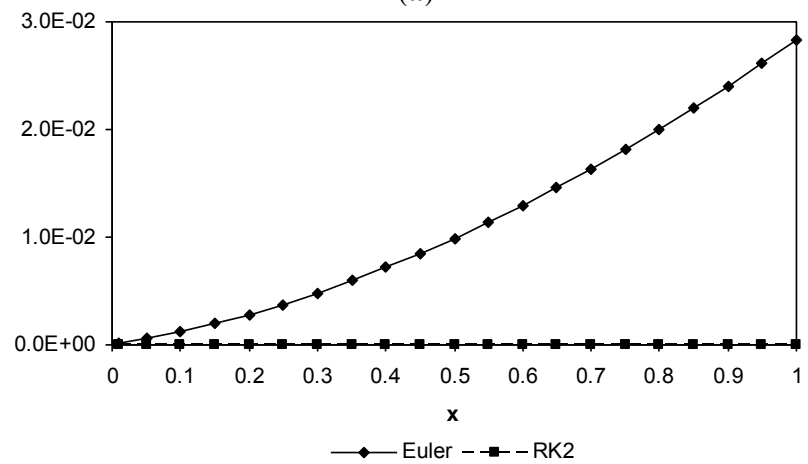

(b)

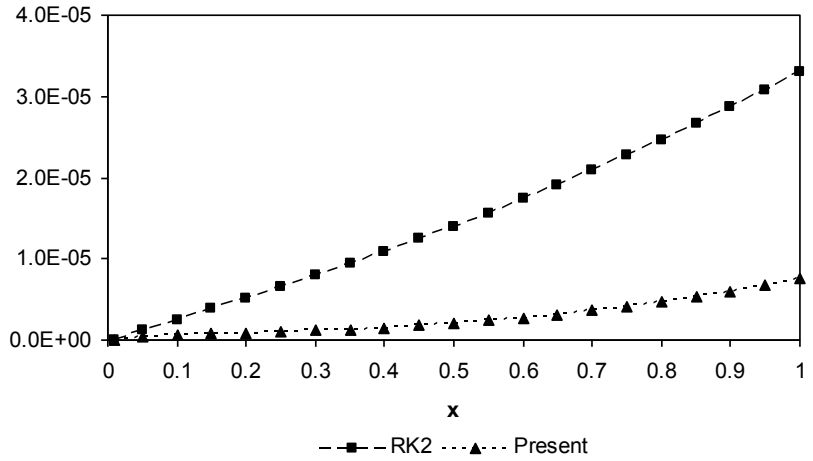

Fig. (2). The absolute error of the RK2 and the Euler methods in (a) and the present and the RK2 in (b) with $h=0.01$.

$y^{\prime \prime}+\frac{2}{x} y^{\prime}+y=6+12 x+x^{2}+x^{3} ; 0<x \leq 1, y(0)=0, y^{\prime}(0)=0$,

with the exact solution $y=x^{2}+x^{3}$. The results of the error for the second order implicit Runge-Kutta (RK2) and the implicit Euler methods are plotted in Fig. (2a) and the present and the second order implicit Runge-Kutta (RK2) methods are plotted in Fig. (2b).

Example 2: Consider a second order nonlinear, nonhomogeneous Lane-Emden equation [9]

$y^{\prime \prime}+\frac{2}{x} y^{\prime}+y^{3}=6+x^{6} ; \quad 0<x \leq 1, \quad y(0)=0, y^{\prime}(0)=0$

(a)

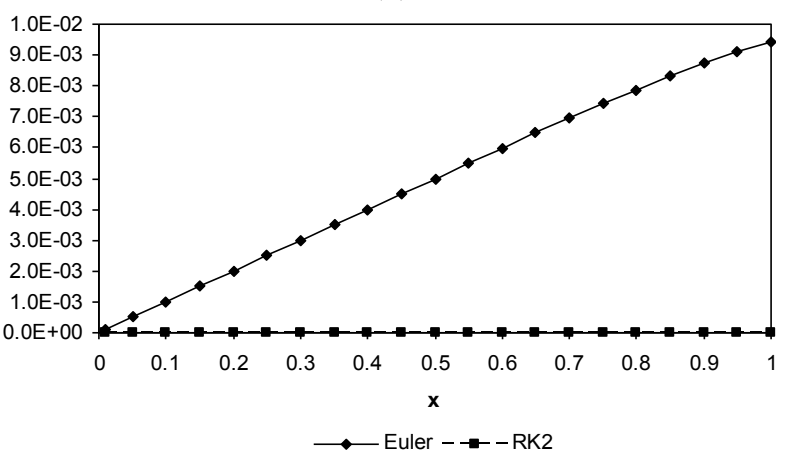

with the exact solution $y=x^{2}$. The results of the error for the second order implicit Runge-Kutta (RK2) and the implicit Euler methods are plotted in Fig. (3a) and the present and the second order implicit Runge-Kutta (RK2) methods are plotted in Fig. (3b).

Example 3: Consider a second order linear, nonhomogeneous Emden-Fowler equation [16]

$$
\begin{aligned}
& y^{\prime \prime}+\frac{8}{x} y^{\prime}+x y=x^{5}-x^{4}+44 x^{2}-30 x ; \\
& 0<x \leq 1, \quad y(0)=0, y^{\prime}(0)=0
\end{aligned}
$$

with the exact solution $y=x^{4}-x^{3}$. The results of the error for the second order implicit Runge-Kutta (RK2) and the

(b)

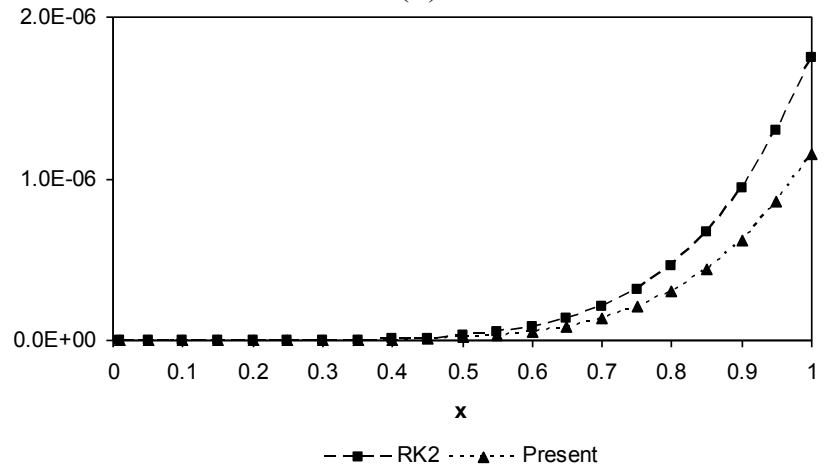

Fig. (3). The absolute error of the RK2 and the Euler methods in (a) and the present and the RK2 in (b) with $h=0.01$.

(a)

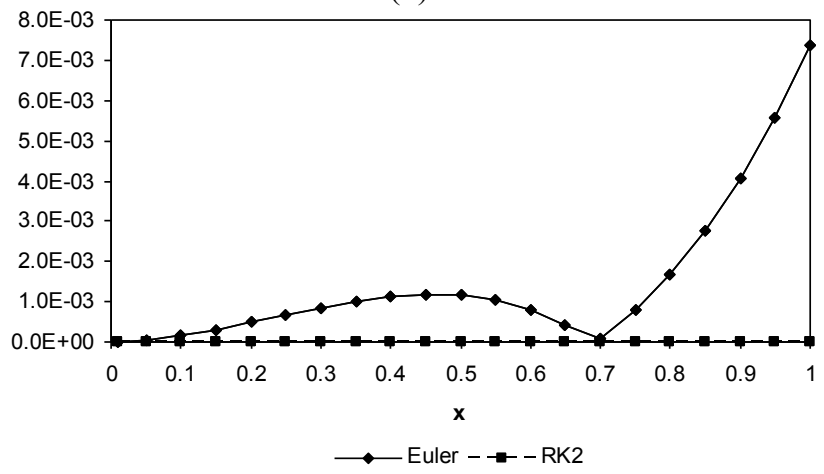

(b)

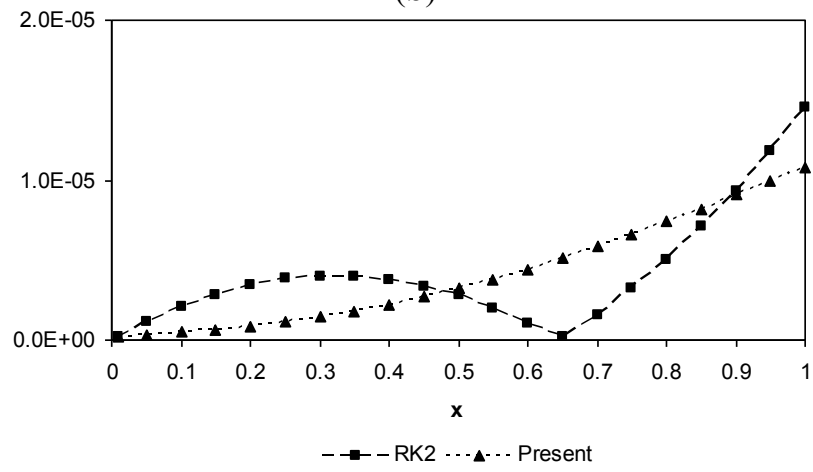

Fig. (4). The absolute error of the RK2 and the Euler methods in (a) and the present and the RK2 in (b) with $h=0.01$. 
implicit Euler methods are plotted in Fig. (4a) and the present and the second order implicit Runge-Kutta (RK2) methods are plotted in Fig. (4b).

Example 4: Consider a second order nonlinear, homogeneous Lane-Emden equation [15]

$y^{\prime \prime}+\frac{2}{x} y^{\prime}+y^{1.5}=0 ; \quad 0<x \leq 1, \quad y(0)=1, y^{\prime}(0)=0$,

with the approximate exact solution $y=\exp \left(-x^{2} / 6\right)$. The results of the error for the present, the second order implicit Runge-Kutta (RK2) and the implicit Euler methods are plotted in Fig. (5).

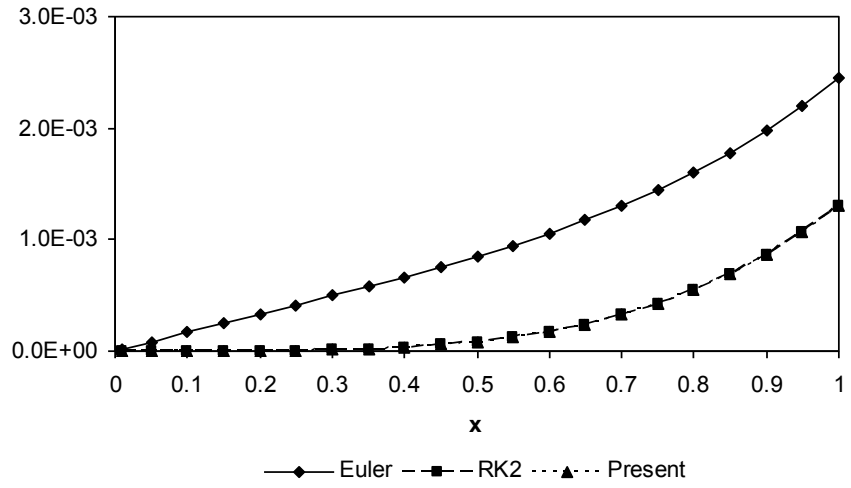

Fig. (5). The absolute error of the present, the RK2 and the Euler methods with $h=0.01$.

\section{RESULTS AND DISCUSSION}

An implicit method has been presented to solve second order singular initial value problems. To illustrate the method, the approximate solutions of some homogenous and non-homogeneous equations have been compared with their exact solutions. First a linear non-homogeneous Eq. (12) having a singular point at $x=0$ has been considered. The approximate solution of Eq. (12) has been obtained by the formula Eq. (4) and the error has been presented in Fig. (2) together with corresponding errors of second order implicit Runge-Kutta and implicit Euler methods. Fig. (2) shows that the error of the present method is smaller than those obtained by second order implicit Runge-Kutta and implicit Euler methods.

Then a non-linear non-homogeneous Eq. (13) has been considered. In this case the error has been presented in Fig. (3) together with corresponding errors of second order implicit Runge-Kutta and implicit Euler methods. This figure indicates that the present method also provides better results than those obtained by second order implicit Runge-Kutta and implicit Euler methods.

Next, a linear non-homogeneous Eq. (14) has been considered and the errors eventually found through the above three methods have been shown in Fig. (4). It is obvious from the Fig. (4) that the errors by the present method increase gradually. But the errors of the second order implicit Runge-Kutta and implicit Euler methods increase rapidly after a short interval.

Finally, a non-linear homogeneous Eq. (15) has been considered and the errors of the above three methods are presented in Fig. (5). It indicates that the error of the present

method is smaller than that obtained by implicit Euler method. In this case the present solution is very close to that obtained by second order implicit Runge-Kutta method.

Based on these above observations, it is concluded that the present method (i.e., the formula Eq. (4)) is more suitable than some existing classical methods for solving a class of equations presented in Eq. (7).

\section{CONFLICT OF INTEREST}

The authors confirm that this article content has no conflict of interest.

\section{ACKNOWLEDGEMENTS}

The authors are grateful to the honorable reviewers for their constructive comments to improve the quality of this article. The authors are also grateful to Mr. Md. Sakhawat Hossain, Assistant Professor, Department of English, Rajshahi University, for his assistance to prepare the revised manuscript.

\section{REFERENCES}

[1] Chandrasekhar S. Introduction to the study of stellar structure. Dover: New York 1967.

[2] Davis HT. Introduction to nonlinear differential and integral equations. Dover: New York 1962.

[3] Richardson OU. The emission of electricity from hot bodies. London: Longmans, Green \& Co. 1921.

[4] Wazwaz AM. A new algorithm for solving differential equations of Lane-Emden type. Appl Math Comput 2001; 118: 287-310.

[5] Wazwaz AM. A new method for solving singular initial value problems in the second- order ordinary differential equations. Appl Math Comput 2002; 28: 45-57.

[6] Wazwaz AM. The modified decomposition method for analytic treatment of differential equations. Appl Math Comput 2006; 173: $165-76$.

[7] Wazwaz AM. Adomian decomposition method for a reliable treatment of the Emden-Fowler equation. Appl Math Comput 2005; 61: 543-60.

[8] Hasan YQ, Zhu LM. Solving singular initial value problems in the second-order ordinary differential equations. J Appl Sci 2007; 7(17): 2505-8.

[9] Hasan YQ, Zhu LM. Modified Adomian decomposition method for singular initial value problems in the second-order ordinary differential equations. Surv Math Appl 2008; 3: 183-93.

[10] Gupta VG, Sharma P. Solving singular initial value problems of Emden-Fowler and Lane- Amden type. Int J Appl Math Comput 2009; 1(4): 206-12.

[11] Demir H, Süngü İC. Numerical solution of a class of nonlinear Emden-Fowler equations by using differential transform method. J Arts Sci 2009;12/Aralı: 75-82.

[12] Mukherjee S, Roy B, Chaterjee PK. Solution of Lane-Emden Equation by differential transform method. Int $\mathrm{J}$ Nonlinear Sci 2011; 12(4): 478-84.

[13] Koch O, Kofler P, Weinmuller E. The Implicit Euler method for the numerical solution of singular initial value problems. Appl Numer Math 2000; 34: 231-52.

[14] Koch O, Weinmuller E. Analytic and numerical treatment of a singular initial value problem in avalanche modeling. Appl Math Comput 2004; 148: 561-70.

[15] Benko D, Biles DC. Robinson MP. Spraker JS. Numerical approximation for singular second order differential equations. Math Comput Model 2009; 49: 1109-14.

[16] Lakestani M, Saray BN. Numerical solution of singular IVPs of Emden-Fowler type using Legendre scaling functions. Int $\mathrm{J}$ Nonlinear Sci 2012; 13(2): 211-9. 
[17] Hasan MK, Ahamed MS, Alam MS, Hossain MB. An implicit method for numerical solution of singular and stiff initial value problems. J Comput Eng 2013; Article ID 720812: 1-5.

[18] Huq MA, Hasan MK, Rahman MM, Alam MS. A simple and straightforward method for evaluating some singular integrals. Far East J Math Educ 2011; 7(2): 93-107.
[19] Hasan MK, Huq MA, Rahman MS, Rahman MM, Alam MS. A new implicit method for numerical solution of singular initial value problems. Int J Concept Comput Info Technol 2014; 2(1): 87-91.

(C) Hasan et al.; Licensee Bentham Open.

This is an open access article licensed under the terms of the Creative Commons Attribution Non-Commercial License (http://creativecommons.org/licenses/by-nc/

3.0/) which permits unrestricted, non-commercial use, distribution and reproduction in any medium, provided the work is properly cited. 